\title{
¿CÓMO PERCIBEN LAS INSTITUCIONES PÚBLICAS Y PRIVADAS A LAS UNIVERSIDADES EN EL FOMENTO DEL EMPRENDIMIENTO?
}

\author{
Mg. Emma Pérez Palacios* \\ eeperezpalacios@yahoo.es
}

\begin{abstract}
RESUMEN
El presente artículo muestra la segunda parte de los resultados del estudio "Fomento del emprendimiento en los jóvenes universitarios 2010". Este estudio recoge los aportes de 9 centros de formación superior y 5 instituciones públicas y privadas vinculadas al fomento del emprendimiento. En esta oportunidad, presentamos la posición de expertos y entendidos en el fomento del emprendimiento de instituciones públicas y privadas respecto al rol de las universidades en esta noble labor.

Según este estudio, fomentar emprendimiento es importante en el proceso de formación de los jóvenes universitarios; a pesar de esta clasificación, las instituciones públicas y privadas consideran que los centros de formación superior no están viendo en el fomento del emprendimiento un camino válido para la realización personal y profesional de los jóvenes.

Palabras claves: emprendimiento, cultura emprendedora, la universidad y la creación de empresas.

\section{ABSTRACT}

This paper presents the second part of the study results "Promoting Entrepreneurship in the University Youth 2010" This study reflects the inputs of 9 higher education establishments and 5 public and private institutions linked to the promotion of entrepreneurship. This time we present the position of scholars and practitioners in the promotion of entrepreneurship of public and private institutions regarding the role of universities in this noble work.

Based on this study, encourage entrepreneurship is important in the process of training of university students, despite this classification, the public and private institutions believe that higher education institutions are not seeing in promoting entrepreneurship a valid path for the realization professional staff and youth.
\end{abstract}

Keywords: Entrepreneurship, entrepreneurial culture, academia and business creation.

\footnotetext{
* Magíster en administración, Licenciada en estadística, Docente de la Facultad de Ciencias Administrativas de la UNMSM.
} 


\section{INTRODUCCIÓN}

¿Qué tan importante es el fomento del emprendimiento desde las universidades para la sociedad? Una respuesta válida podemos encontrarla en la experiencia mexicana. En México, existen 114 incubadoras de empresas enfocadas en el público universitario. De ellas, 63 están en universidades tecnológicas, 47 en institutos tecnológicos y 4 en universidades politécnicas.

El objetivo de esta enseñanza es, por un lado, evitar que los emprendedores no tengan impulsos para materializar sus ideas y, por otro, evitar que sus empresas mueran antes de los dos años, promedio de vida de una pyme en México.

¿Cuáles son los resultados de estos apoyos universitarios? En México se han creado más de 2.500 nuevas empresas, (micro, pequeñas y medianas), que han conseguido dar trabajo a un total de 7.500 personas. Las pymes mexicanas han encontrado en las incubadoras universitarias un gran trampolín para el competitivo de un sector emergente.

¿Y en Perú? ¿Cómo está avanzando el fomento del emprendimiento? A continuación expondremos cuáles son -a juicio de los expertos- las principales limitaciones de la actividad emprendedora y su impacto en el desarrollo del país; así mismo se analizará cómo influye la percepción del futuro del país en la creación de empresas sostenibles y exitosas.

\section{MÉTODOS}

Como se explicó en la primera entrega de los resultados del estudio, la investigación realizada recoge información cualitativa. Para ello, se usó la la técnica de la entrevista, a través de cuestionarios estructurados dirigidos a dos grupos de discusión claramente identificados y diferenciados:

Grupo 1: Representantes de 9 centros de formación superior (universidades e institutos).

Grupo 2: Representantes de 5 instituciones públicas y privadas comprometidas con el fomento del emprendimiento.

Así también, se clasifica como una investigación del tipo descriptiva, porque describe en qué medida los centros de formación superior están fomentando la cultura emprendedora de sus estudiantes y cómo, a decir de los expertos y entendidos en el fomento del emprendimiento, perciben la labor de las universidades en esta no- ble labor. Es preciso destacar que en el presente artículo se muestran los resultados obtenidos solo del grupo 2.

\section{RESULTADOS}

1. Importancia del fomento del emprendimiento: Los representantes de las instituciones públicas y privadas consideran que:

- Es importante fomentar en jóvenes el emprendimiento empresarial y la creación de empresas no sólo desde los centros de formación superior, sino en todos los niveles educativos empezando en la educación inicial.

- Los emprendedores por lo general "no salen" de las universidades, por lo que es esencial desarrollar el espíritu emprendedor entre los docentes, para luego trabajar y transmitir ese espíritu a los estudiantes.

- Las instituciones educativas tienen la responsabilidad de fortalecer capacidades emprendedoras en los estudiantes, ampliándoles su visión de plan de vida.

2. Nivel de preparación y motivación de los estudiantes de formación superior para iniciar su propia empresa: Las instituciones públicas y privadas consideran que:

- Los jóvenes saben elaborar planes de negocio, es decir, están preparados técnicamente para desarrollar buenos proyectos.

- Sin embargo, los jóvenes no están interesados en llevar adelante sus proyectos, y eso es lo que marca la diferencia a favor de un emprendedor.

- La causa principal para identificar esta actitud en los jóvenes es la percepción del temor a los cambios y el miedo al fracaso.

3. Importancia del apoyo de los centros de formación superior en la promoción del emprendimiento:

- Es muy importante porque las empresas son el vehículo para el crecimiento del país, además porque es fundamental juntar los conocimientos profesionales con las capacidades emprendedoras que permitan crear empresas sostenibles en el tiempo, en suma, porque las universidades tienen las herramientas necesarias para brindarle sostenibilidad y transferencia tecnológica a las empresas. 
4. Respecto a las acciones que realizan las instituciones públicas y privadas para la promoción del emprendimiento y la creación de empresas, estas son:

- Programas de capacitación y talleres.

- Convenios para la ejecución de concursos de planes de negocios.

- Ejecución directa de concursos de ideas de negocios y planes de negocio.

- fomento de incubadoras de empresas.

- Conferencias.

- Edición de publicaciones.

- Programa radial sobre emprendimientos.

- Facilitación de acceso a la información a través de la Internet.

- Apoyar en algunos casos con capital semilla.

- Diseñar y transferir metodologías participativas para las fases de motivación y preparación (conocimiento) de emprendedores.

5. El rol del Estado en la promoción de la actividad emprendedora y la creación de empresas: Las instituciones públicas y privadas opinan que el Estado:

- Debe asignar recursos para el apoyo al fomento del emprendimiento.

- Se debe mejorar el Poder Judicial, esto garantizaría una rápida adecuada administración de justicia.

- Debe cumplir un rol promotor más activo, a fin de garantizar las mejores condiciones para el nacimiento de nuevas empresas.

- Debe desarrollar instrumentos válidos y transferir metodologías para que se implementen programas de apoyo

6. Fases por las que debe transcurrir el fomento del emprendimiento desde los centros de formación superior. Según la experiencia de los expertos consultados, las universidades e institutos deben al menos trabajar en tres fases o etapas para fomentar el espíritu emprendedor:

- Fase 1. Identificación de ideas de negocio y fortalecimiento de capacidades emprendedoras; se debe asistir al estudiante en la búsqueda de ideas de negocio orientadas al mercado, asimismo, y de manera paralela a todo el proceso, se debe trabajar en el desarrollo y/o fortalecimiento de su actitud emprendedora, haciéndole ganar en confianza y seguridad sobre sí mismo y sobre el proyecto. Esta fase se refiere a la parte motivacional.

- Fase 2. Elaboración del Plan de Negocios; es el apoyo en toda la parte técnica y preparatoria de la nueva empresa, se trata de recoger la mayor información posible a fin de reducir el riesgo del negocio. Esta fase se refiere a la parte de preparación o conocimientos.

- Fase 3. Sostenibilidad en la implementación del Nuevo Negocio; es la etapa de inicio del negocio, n la que el emprendedor se lanza al mercado con su iniciativa empresarial. En esta etapa requiere de sus primeros clientes, asistencia en la parte contable, administrativa, en el proceso de producción, en mejorar y/o fortalecer la imagen del producto, servicio o de la misma empresa. Se refiere a la etapa de puesta en marcha del proyecto.

7. Principales razones por las que el joven universitario no se ve atraído por el desafío emprendedor. A decir de los representantes de las instituciones públicas y privadas, son (en orden de importancia):

- Desconocimiento de la gestión de negocios.

- Desconocimiento del mercado a atender.

- Falta de capital.

- Poca experiencia en el rubro.

\section{CONCLUSIONES}

1. Las instituciones públicas y privadas reconocen la importancia de fomentar el emprendimiento desde el sistema educativo superior, pero llama la atención que las acciones que se vienen realizando entre unas y otras no estén articuladas a fin de lograr los objetivos comunes.

2. El sistema universitario tiene aún una escasa contribución al fomento de la actividad emprendedora.

3. Si bien es cierto, la mayoría de estudiantes universitarios tienen los conocimientos técnicos para desarrollar un proyecto empresarial, estos mismos jóvenes no tienen el nivel de motivación necesario como para asegurar la inclinación por el emprendimiento 
como opción de realización personal y profesional.

4. A pesar de los esfuerzos, la universidad no está alentando la formación de líderes emprendedores, está concentrada en formar "empleados" capaces de administrar empresas, de contribuir al sector público y privado con profesionales dispuestos a recibir órdenes de un superior. En este contexto, lo que resulta fundamental es tener clara la idea que "los empleados buscan trabajo después que se construye el negocio y que el trabajo del emprendedor comienza antes del negocio". Por lo tanto, es urgente empezar a formar emprendedores que contribuyan al desarrollo económico y social a nivel local, regional y nacional, a través de la creación de nuevas empresas.

5. Es fundamental fortalecer las redes de apoyo entre la universidad y las instituciones públicas y privadas que promueven el emprendimiento, de este modo se podría suplir las carencias o desconocimientos respecto al tema promocional.

\section{BIBLIOGRAFÍA}

Sistema Nacional de Incubadoras de Empresas de México - SNIE, Secretaría de Economía 2012.
Linares, Dino - Colectivo Integral de Desarrollo, en estudio elaborado para el Ministerio de Trabajo y Promoción del Empleo: "Creación de Empresas y apoyo a Emprendedores en el Perú", Lima 2002.

Dirección Nacional de la Micro y Pequeña Empresa - Ministerio de Trabajo y Promoción del Empleo, Elaboración de Estadísticas de la Micro y Pequeña Empresa, 2006

Dirección Nacional de la Micro y Pequeña Empresa - Ministerio de Trabajo y Promoción del Empleo, Directiva № 001-2008-MTPE/3.11.3, Ejecución de acciones para el fomento del emprendimiento con enfoque de oportunidad a nivel regional, 2008

Asociación Peruana de Incubadoras de Empresas, Perú Incuba www.peruincuba.net

Sub Secretaría de PYME - Secretaría de Economía del Estado de México, Sistema Nacional de Incubadoras de Empresas, SNIE www.contactopyme.gob.mx/snie/PrinciplSNIE.asp

Tefarikis, Elías: Revista América Economía Artículo "Educación para el emprendimiento - Chile”, México 2009

Wompner, Fredy: "Un modelo de incubadora de negocios universitaria; en la ruta de la innovación organizacional”, Chile 2007. 